Recent Advances in Communication, Electronics \& Electrical Engineering

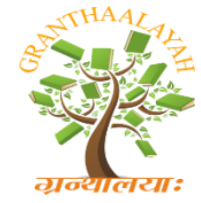

INTERNATIONAL JOURNAL OF RESEARCH GRANTHAALAYAH

A knowledge Repository

RACEEE - 17

\title{
ROLE OF EQUIPMENT MANAGEMENT IN OPTICAL TRANSPORT NETWORK
}

\author{
Divya Jha ${ }^{* 1}$, Bimlendu Shahi ${ }^{2}$, Dr. Suresh $D^{3}$, Dr. Dushyanth $N^{4}$ \\ ${ }^{* 1,2,3,4}$ Electronics and Communication, Jain University, India
}

DOI: https://doi.org/10.29121/granthaalayah.v5.i4RACEEE.2017.3338

\begin{abstract}
The ever-developing interest of present day society for information with enhanced execution over longer separations have made the system complex in terms of architecture, technologies, Network Elements, communication between Network Elements, security and signaling control. Equipment Management as a segment of Network Management System gives administrators the capacity to control general operation of the NEs, superintend interaction among NEs and to upper layer administration framework, thus automating the task of managing complex network. This paper presents the role of Equipment Management in Optical Transport Network and some of its challenges.

Keywords: Equipment Management; Network Elements; Optical Transport Network.

Cite This Article: Divya Jha, Bimlendu Shahi, Dr. Suresh D, and Dr. Dushyanth N D. (2017). "ROLE OF EQUIPMENT MANAGEMENT IN OPTICAL TRANSPORT NETWORK." International Journal of Research - Granthaalayah, 5(4) RACEEE, 155-160. https://doi.org/10.29121/granthaalayah.v5.i4RACEEE.2017.3338.
\end{abstract}

\section{Introduction}

Optical Network has emanated as the major transport network which has exceptionally advanced in terms of speed and in addition reaches of 100's of $\mathrm{Gb} / \mathrm{s}$ transmission speed per wavelength, and several kilometers of signal transmission with flexible spectrum distribution and allocation and channel sharing in frequency and time domains while eliminating the need of regeneration [1]. 
Recent Advances in Communication, Electronics \& Electrical Engineering

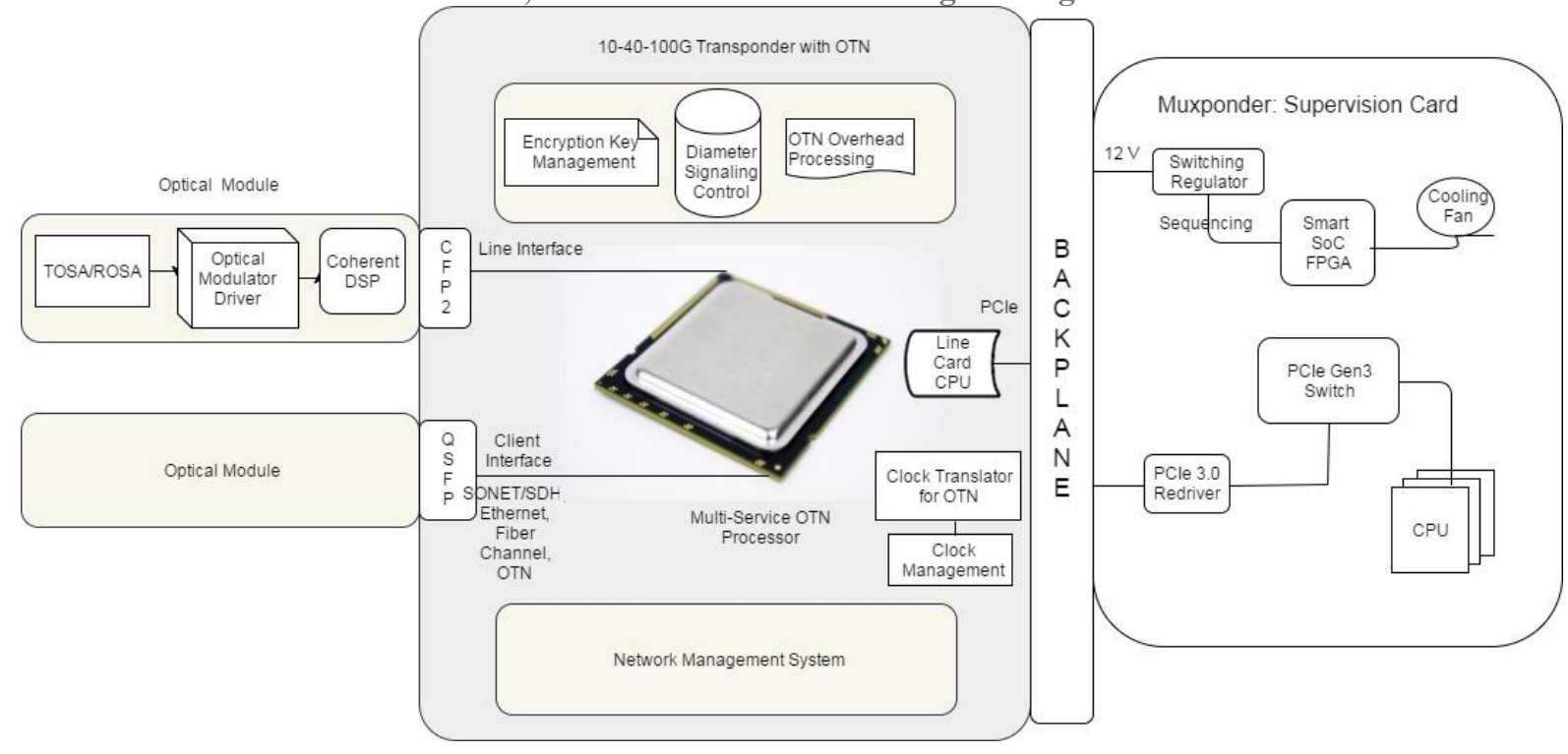

Figure 1: Logical Configuration of Optical Transport Network

Fig 1 depicts basic logical arrangement of Optical Transport Network. It comprises of Network Elements which forms the core of OTN and is a manageable logical unit which contains one or more physical equipment [2]. Optical module intercepts optical signal, interfaces to MultiService OTN digital signal processor which delivers processed signal again back to the optical module. In order to accumulate multiple services onto single wavelength and then multiplex this wavelength with other wavelengths into a single fiber, Muxponder is used in optical networking. Muxponder incorporate multiple high processing CPUs, switches and drivers, each of them requiring proper management [3].

The evolvement of different technologies as well as the networking devices have played major role in advancement of OTN. With the advancement of OTN, the complexity of the distributed network has increased in terms of equipment, interaction among devices, signaling control and security. Conventional management and control planes for fiber optic systems are also developing to fulfill the prerequisites of diverse traffic and services, while utilizing separate as well as shared infrastructures [4]. The networking system has been structured to integrate newer technologies improving its ability to deliver and accomplish the ever-increasing demand of data from enterprise and global community.

At least three diverse activities have to be performed by every single network element: Process and evaluate transit traffic in data plane; determine the interaction of the devices based on processed signal with control plane protocols and interact with the owner i.e; Network Management System(NMS) through management plane. Various parameters that need to be considered at the management plane include [5]

- Connection set-up time, which could range from fraction of second to days. This depends on the demand of service and client characteristics, 
Recent Advances in Communication, Electronics \& Electrical Engineering

- Service Availability and Resilience which can include Service mean down time, Out of service state, recovery time with degradation in quality and performance or complete service recovery time.

- Routing Constraints: Routing Stability, which must be provided without affecting QoS parameters such as jitter, throughput, delay and loss; Routing Differentiation which involves physical path differentiation.

- Confidentiality: Level of secrecy to be maintained for a given service. Includes encryption key management and Diameter Signaling Control (DSC).

- Distance: Physical distance, which is generally considered for billing purpose.

Equipment Management System(EMS) manages and deals with the functionality and capabilities within each NE. This management system supports the traffic between itself and other NEs and delivers the information upward to higher level management system via CORBA interface. Fig 2 depicts the position of EMS in Network Management Architecture. The network today is based on varieties of different technologies and is designed for integrated access, high speed data/ video, transport and switching of voice. Since each network element has complexity, it is accompanied by an Equipment Management system which exploits the power of technology while concealing its complexity.

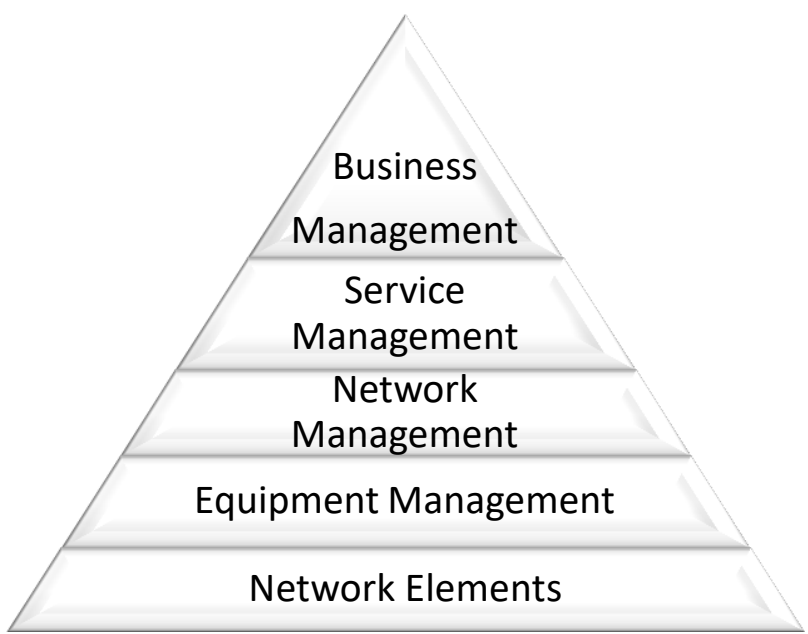

Figure 2: Network Management Architecture

The purpose of EMS is network deployment and planning, network inventory management, network provisioning, monitoring, service provisioning, service assurance, maintenance, overhaul and control.

\section{Literature Review}

Xinyi et al. [6], have proposed an embedded network management architecture with web mode in Linux and Simple Network Management Protocol Agent. This method demonstrates the network management on a small scale which can further be extended to develop an enterprise network. Robert D. Doverspike et al. [7] discusses optical network control, management and 
Recent Advances in Communication, Electronics \& Electrical Engineering operation for a large telecommunication network with DWDM equipment. Similar concept can be applied to the telecommunication network supporting other technologies such as SWDM, OCS, OPS, PON etc.

Scott Daniel Wilsey et al. [8] have presented a template configuration based management system which utilizes configuration information from the network element. The element manager and programming acquire at least one of the configuration parameter values which is not similar to the corresponding configuration parameter value in the template configuration. Based on this determination, the element manager and programming take an action. This method is found to have less processing overhead.

Further, Gary Faulker et al. [9] have presented a network monitoring system. This system includes a SNMP enabled communications interface, at least one device definition file and data access interface. The included definition file contains query commands related to Management Information Bases (MIBs) associated with a network device. The SNMP enabled communication interface may get an access to the network device using query commands and may obtain response data from the network device. For external access to the received data, the data access interface and threshold may be configured. This system can further be extended to provide configuration parameters such as signal rate, monitoring network elements and troubleshooting in case of any fault. Further, both the management systems' usability can greatly be enhanced with development of user-friendly environment.

Bin Lu et al. [10], demonstrated an embedded element management system supported with userfriendly GUI (Graphical User Interface) and I/Os, which enables administrators to configure and monitor the managed equipment in the most convenient manner.

David Jordan et al. [11] have presented Operations, Administration, Maintenance, and Provisioning (OAM\&P) system associated with the network element, wherein the main processor is accessible through at least one access techniques; a supervisory plane consisting of a secure memory and a secure processor wherein the supervisory plane is isolated from and communicatively coupled to the main memory and the main processor. Supervisory plane is configured to permit direct and secure access to the main memory and the main processor. This system provides a secure access to the network elements to the Administrator for proper maintenance but still lacks a secure access system for the users. The management system developed can further be enhanced by providing appropriate user accessibility to the network elements which can help to improve Equipment Manager usability.

G. Cordray et al. [12] have presented network management applications and policy that are adopted and communicated automatically upon the identification of new device or application or protocol in the network. The authors have also presented that at least one aspect of the network information is obtained by an NMAS, and at least one of the management policies that is applicable is identified by the NMAS; and such identified policy is then used to manage at least one of the aspects of the network operation. 
Recent Advances in Communication, Electronics \& Electrical Engineering

\section{Challenges}

As the newer technologies are appearing to keep up with the ever-increasing bandwidth requirement of the world, service providers have the objective to congregate towards a unified network in order to render differentiated services in a single network and aid divergent requirements of various clients. With this, network has grown to be complex than ever and there is a need of automation of configuration and management tasks. Each network element deployed to provision higher speed and reach have different parameters. Automatically detecting, integrating and managing the newly evolved network elements and further monitoring the status of each entity to provision uninterrupted service is the present challenge of Equipment Management System. Further, there is the need of security of newly deployed equipment and also the security of signals. Automatic selection of encryption and signaling control mechanism and policy for the security of Network Element and the path created in the network is another challenge of EMS.

\section{Conclusions}

Equipment Management System plays a major role in managing the network elements and thus the network for provisioning of high speed integrated services. Over the time network has changed and so has the Equipment Management System. The management technique presented by G. Cordray et al. for non-traditional and advanced network and information system topologies which automatically adopts and communicates network management application and policy upon detection of a new device, application or protocol in the network has found to be the most appropriate. The Equipment Management System can further be enhanced by creating a hierarchical accessibility with different level of privilege. The system's usability can be improvised by decentralizing the monitoring function wherein users can be given appropriate accessibility to monitor the health status of the Network Elements connected to hub site. Security is one of the major concerns while creating a hierarchical level for providing accessibility to users. Appropriate encryption mechanism and signaling control can be applied to each existing and newly deployed Network Elements.

\section{References}

[1] AG Young, S Gareau, T Norman, R Wyatt," Optical transport network clock transient suppression systems and methods", US 9264139 B2 (2016).

[2] M Schulz, J Maurer," Method for operating a remote controlled network element", US 9282002 B2 (2016).

[3] M Dzida, A Bak - Recent Advances in Computational Optimization," Flow Design and Evaluation in Photonic Data Transport Network", Recent Advances in Computational Optimization, Vol 655 (2016).

[4] Admela Jukan and Joe Mambretti, "Evolution of Optical Networking Toward Rich Digital Media Services", Proceedings of the IEEE, 2012.

[5] Wissam Fawaz et al.," Service Level Agreement and Provisioning in Optical Networks", MANAGEMENT OF OPTICAL NETWORKS, IEEE Communications Magazine (2004).

[6] Xinyi $\mathrm{Wu}$, Yong Zhu, Xiaolong Deng, "Design and Implementation of Embedded SNMP Network Management Manager in Web-based Mode", IEEE Asia-Pacific Services (2008). 
Recent Advances in Communication, Electronics \& Electrical Engineering

[7] Robert D. Doverspike, Jennifer Yates, “Optical Network Management and Control”, Proceedings of the IEEE (2012).

[8] Scott Daniel Wilsey, Darren William Oye," Managing a network element using a template configuration “, US 7720940 B1 (2010).

[9] Gary S. Miliefsky,” Enterprise security management for network equipment “, US 20090199298 A1 (2009).

[10] Bin Lu, Yangan Zhang, Jinnan Zhang, "Design and Implementation of New Embedded Network Management and Monitoring System", Applied Mechanics and Materials Vols 263-266, Trans Tech Publications (2013).

[11] David Jordan Krauss, Stephen B. Alexander, Loudon Thomas Blair," Secure access systems and methods to network elements operating in a network", US 9503443 B2(2016).

[12] David F. Link, Christopher G. Cordray, Richard M. Chart, Karl Ginter, "Management techniques for non-traditional network and information system topologies "US 9537731 B2,(2017).

*Corresponding author.

E-mail address: divyajha73@yahoo.com 